ARTICLE

DOI: $10.1038 / \mathrm{s} 41467-018-05396-x$

\title{
Block copolymer crystalsomes with an ultrathin shell to extend blood circulation time
}

Hao Qi (1) 1, Hao Zhou (1) 1, Qiyun Tang (1) 2, Jee Young Lee ${ }^{3}$, Zhiyuan Fan', Seyong Kim¹, Mark C. Staub ${ }^{1}$,

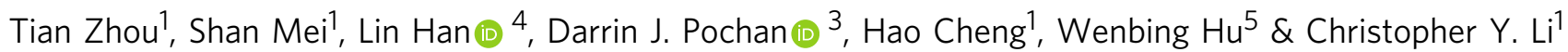

In water, amphiphilic block copolymers (BCPs) can self-assemble into various micelle structures depicting curved liquid/liquid interface. Crystallization, which is incommensurate with this curved space, often leads to defect accumulation and renders the structures leaky, undermining their potential biomedical applications. Herein we report using an emulsionsolution crystallization method to control the crystallization of an amphiphilic BCP, poly (L-lactide acid)-b-poly (ethylene glycol) (PLLA-b-PEG), at curved liquid/liquid interface. The resultant $B C P$ crystalsomes (BCCs) structurally mimic the classical polymersomes and liposomes yet mechanically are more robust thanks to the single crystal-like crystalline PLLA shell. In blood circulation and biodistribution experiments, fluorophore-loaded BCCs show a $24 \mathrm{~h}$ circulation half-life and a $8 \%$ particle retention in the blood even at $96 \mathrm{~h}$ post injection. We further demonstrate that this good performance can be attributed to controlled polymer crystallization and the unique BCC nanostructure.

\footnotetext{
${ }^{1}$ Department of Materials Science and Engineering, Drexel University, Philadelphia, PA 19104, USA. ${ }^{2}$ Institut für Theoretische Physik, Universität Göttingen Friedrich-Hund-Platz 1, 37077 Göttingen, Germany. ${ }^{3}$ Department of Materials Science and Engineering, University of Delaware, Newark, DE 19716, USA. ${ }^{4}$ School of Biomedical Engineering, Science \& Health Systems, Drexel University, Philadelphia, PA 19104, USA. ${ }^{5}$ Department of Polymer Science and Engineering, State Key Lab of Coordination Chemistry, School of Chemistry and Chemical Engineering, Nanjing University, 210023 Nanjing, China. These authors contributed equally: Hao Qi, Hao Zhou. Correspondence and requests for materials should be addressed to H.C. (email: hcheng@drexel.edu) or to C.Y.L. (email: chrisli@drexel.edu)
} 
O ver the past few decades, many elegant delivery systems emerged to mimic biological structures such as lipid membranes and virus capsid ${ }^{1-3}$. For example, red blood cells (RBCs)-mimicking particles were reported to resemble natural RBCs' size, shape, and deformability ${ }^{4-6}$. Self-assembled liposomes that mimic envelope viral structures were developed as tumor-targeting gene delivery carriers ${ }^{7,8}$. Like lipids, synthetic amphiphilic block copolymers (BCPs) can also self-assemble into similar vesicle structures (defined as polymersomes to emphasize its synthetic polymer origin) in water ${ }^{9,10}$. Because of the high molecular weight and chain entanglement of the hydrophobic blocks, polymer vesicles are mechanically more stable than liposomes ${ }^{10}$. Yet polymersomes' membranes are flexible, and their morphologies/shapes may change over time, particularly under a high shear field. While shape transformation of polymersomes is of great physical and biological interest, in vivo applications call for robust and mechanically stable carrier structures.

Strategies towards permanent BCP assemblies include using crosslinking agents to form shell-cross-linked knedel-like (SCK) particles and incorporating BCP with self-crosslinkable segment ${ }^{11,12}$. Early studies in polymersome systems showed that polymer crystallization affected the formation of polymersomes as well as their mechanical properties ${ }^{13,14}$. Discher et al. demonstrated that, after polymer crystallization, vesicles of semicrystalline poly(ethylene oxide)- $b$-polycaprolactone (PEO- $b$ PCL) are rigid and leaky, which was attributed to the defects formed between the adjacent polymer crystalline domains (grain boundaries $)^{15}$. Since many biometrically relevant amphiphilic BCPs are crystallizable at ambient conditions, it is of importance to investigate polymer crystallization at curved nanospace. From a crystallographic standpoint, curved space is incommensurate with three-dimensional translational symmetry. Recent studies on spherical crystallography showed intriguing packing behavior of colloidal particle at the surface of microscale water droplets ${ }^{16-18}$. Intriguing defects or voids were introduced to these curved crystals depending on the nature of particle-particle interaction as well as the size of the particles and water droplets ${ }^{16-18}$. We recently developed a miniemulsion-solution crystallization method to study confined crystallization at nanometer-sized, curved liquid/liquid interface. Polymer-single crystal-like poly (Llactide) (PLLA) hollow capsules were formed by carefully directing polymer crystallization at curved liquid/liquid interface $^{19}$. Crystalsome was used to describe this unique assembly, emphasizing its crystalline structure and vesicle-like morphology.
While structurally interesting, these PLLA crystalsomes are hydrophobic and cannot be dispersed in water without added surfactants, limiting their potential biomedical applications.

Herein, we report using the emulsion-solution crystallization method to assemble amphiphilic BCP crystalsomes (BCCs). During the crystallization process, the BCP acts both as the surfactant to stabilize oil droplet in water, and as the crystallizable material to form the crystalsome so that the crystallization process is precisely confined at the curved liquid/liquid interface. Nano-sized BCCs are formed with an asymmetric wall comprised of concentric $2.5 \mathrm{~nm}$ PLLA lamellar crystal and a $2 \mathrm{~nm}$ PEG layer. In the crystal, each PLLA chain folds nine times, rendering a uniform PEG brush layer with a precisely controlled grafting density of 0.3 chain $\mathrm{nm}^{-2}$. While the total wall thickness is only $4.5 \pm 0.4 \mathrm{~nm}$ (mean \pm s.d., $n=10$ ), these BCCs show ultra-long circulation time in vivo. We attribute this superb blood circulation performance to the combination of significantly enhanced mechanical properties of the single crystal-like shell and the uniform PEG brush layer. We envisage BCCs lead to a new class of self-assembled nanoparticle delivery systems.

\section{Results}

Morphology and structure of PLLA- $b$-PEG BCCs. In this study, we selected crystalline and biocompatible polymer PLLA as the hydrophobic segment. PEG is chosen as the second block of the BCP as it affords water solubility and stealthability to increase the particle circulation time in the blood ${ }^{20}$. The fabrication process of PLLA- $b$-PEG block copolymer crystalsome is shown in Fig. 1. In brief, PLLA- $b$-PEG is firstly dissolved in toluene at $95^{\circ} \mathrm{C}$. Water is added into the polymer solution and the mixture is ultrasonicated to generate an emulsion, within which the PLLA segments are confined in the toluene droplet while the PEG segments in the water phase. The emulsion is then quenched to $25^{\circ} \mathrm{C}$ for crystallization. In this design, PLLA- $b$-PEG molecules are pinned at the curved liquid/liquid interface after emulsification, crystallization of PLLA is therefore confined at the interface and the formation of more complex spherulites/dendrite crystals inside the droplet is avoided ${ }^{21}$.

Figure $2 \mathrm{a}, \mathrm{b}$ show scanning electron microscopy (SEM) and transmission electron microscopy (TEM) images of the PLLA- $b$ PEG crystalsome that was formed after emulsion-solution crystallization for 9 days. Spherical capsules are seen in both images with a diameter of $\sim 200 \mathrm{~nm}$, which is consistent with

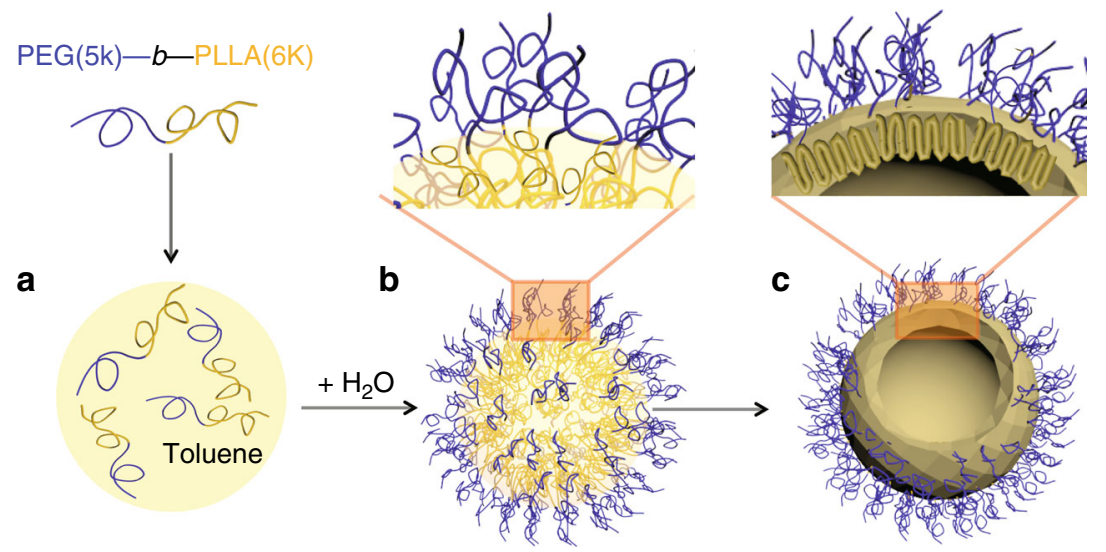

Fig. 1 Fabrication of PLLA-b-PEG block copolymer crystalsomes. a Dissolution of the $\mathrm{BCP}$ in toluene; $\mathbf{b}$ emulsification at $95^{\circ} \mathrm{C}$; $\mathbf{c}$ quenching to $25^{\circ} \mathrm{C}$ for crystallization. The driving force of this assembly process is confined PLLA crystallization at the toluene/water interface, leading to a ninefold PLLA chain conformation as shown in c. This confined crystallization process also leads to a 2.5 nm thick PLLA crystal layer, covered with a precisely controlled, uniform PEG brush layer (grafting density of 0.3 chain $\mathrm{nm}^{-2}$ ) 

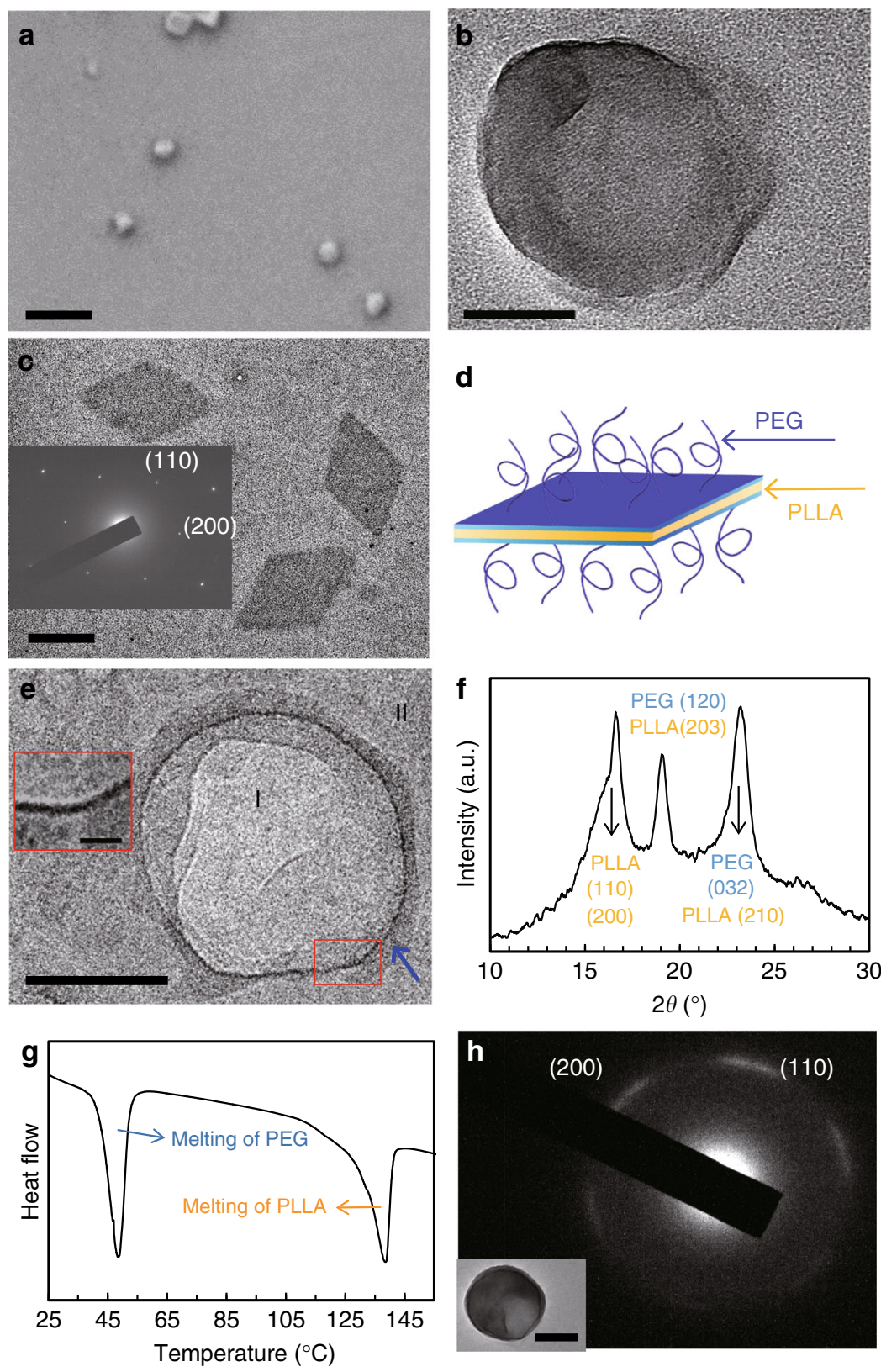

Fig. 2 Morphology and crystal structure of PLLA-b-PEG single crystal and BCCs. a SEM and $\mathbf{b}$ TEM micrographs of BCCs in dried state. $\mathbf{c}$ TEM micrograph of flat PLLA-b-PEG polymer single crystals grown from toluene with an SAED pattern and the corresponding schematics (d). e A representative cryo-TEM micrograph of BCCs in water. Inset of $\mathbf{e}$ is an enlarged image highlighting the BCC wall. $\mathbf{f}$ WAXD pattern, $\mathbf{g}$ a DSC first heating thermogram and $\mathbf{h}$ an SAED pattern of PLLA-b-PEG BCCs. Inset of $\mathbf{h}$ shows a corresponding BCC bright field TEM micrograph. Scale bars in $\mathbf{a}, \mathbf{b}, \mathbf{c}, \mathbf{e}$, inset of $\mathbf{e}, \mathbf{h}$ are 500,100 , $500,50,10$, and $200 \mathrm{~nm}$, respectively

dynamic light scattering (DLS) results (Supplementary Figure 1). Atomic force microscopy (AFM) experiments also confirm the spherical morphology (Supplementary Figure 2). In contrast, PLLA- $b$-PEG single crystals grown from toluene solution instead of toluene/water interface at $25^{\circ} \mathrm{C}$ are flat with a lozenge shape (Fig. 2c), with the PLLA crystalline lamella sandwiched by PEG polymer brushes on both sides. (Fig. $2 \mathrm{~d})^{14}$. The observed spherical capsules in Fig. 2a also appear partially collapsed, which might be due to the sample drying/deposition process. Cryo-TEM imaging of the crystalsome in aqueous solution was then performed to examine the intact morphology of the capsules. As shown in Fig. 2e, the original shape of the PLLA$b$-PEG crystalsome in solution is nearly spherical. Similar electron density in the interior of the capsule (area I) and background (area II) suggests that the crystalsome is hollow. To further prove BCCs are hollow and verify the shell thickness, AFM measurement was conducted on the small crystal pieces after breaking the BCCs by ultrasonication (see later discussion). The spherical morphology is similar to our previously reported homopolymer PLLA crystalsomes ${ }^{19}$. Since PLLA- $b$-PEG is used in the present case, we coin the name BCCs to highlight the copolymer nature of the structure. The morphology and size of the BCCs resemble those of the spherical polymersomes. Figure $2 \mathrm{e}$ also reveals that, unlike most reported polymersomes, these BCCs exhibit relatively rough contour, which is due to polymer crystallization. An enlarged cryo-TEM image of the red box area shows the shell thickness is approximately $2-3 \mathrm{~nm}$, which is attributed to the crystalline PLLA layer. PEG layer is solvated in 

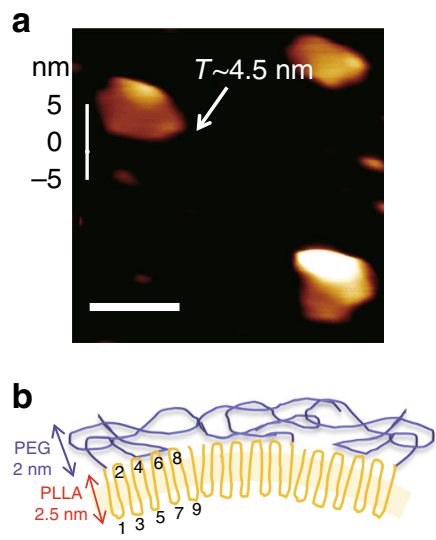

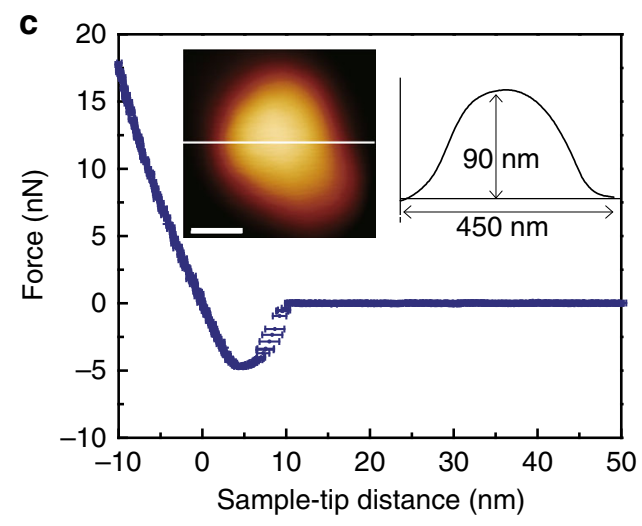

Fig. 3 Mechanical properties of PLLA-b-PEG BCCs. a AFM image and corresponding height profile of a small crystal piece broken using ultrasonication; b Side-view of PLLA- $b$-PEG BCC shell in dried state, showing thickness of individual layer and PLLA chain folding; $\mathbf{c}$ AFM force-deformation spectrum on

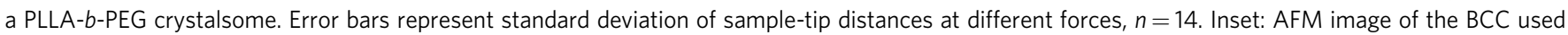
for the indentation test and its height profile. Scale bars are $100 \mathrm{~nm}$

aqueous solution that constitutes the lighter layer on the exterior of the BCCs of $\sim 12 \mathrm{~nm}$, as the blue arrow pointed. The thickness of the PLLA crystalline shell $(2-3 \mathrm{~nm})$ is similar to that of liposomes and much thinner than the hydrophobic core thickness of PLA- $b$-PEG $\left(M_{n}=3.2 \mathrm{k}-2.8 \mathrm{~kg} \mathrm{~mol}^{-1}\right)$ polymersomes (e.g. $\sim 10.4 \pm 1.4 \mathrm{~nm})^{22}$.

To understand the crystalline structure of PLLA, wide angle $\mathrm{X}$-ray diffraction (WAXD) experiments were conducted on dried BCC samples at $25^{\circ} \mathrm{C}$ (Fig. 2f). The WAXD pattern concludes that orthorhombic a form of PLLA is formed ${ }^{19}$. The strongest diffraction peak at $16.7^{\circ}$ is from $(110) /(200)$ planes of PLLA crystals while the peak at $19.8^{\circ}$ is from (203) plane of PLLA and (120) plane of PEG, and the peak at $22^{\circ}-24^{\circ}$ can be attributed to (032) plane of PEG and (210) plane of PLLA ${ }^{19,23}$. Note that the PEG crystals are formed during BCCs solution drying process. Figure $2 \mathrm{~g}$ shows a differential scanning calorimetry (DSC) thermogram of the BCCs, where a heating rate of $10{ }^{\circ} \mathrm{C} \mathrm{min}-1$ was used. The two peaks in the thermogram can be attributed to the melting of PEG (at $49.0^{\circ} \mathrm{C}$ ) and PLLA (at $138.9^{\circ} \mathrm{C}$ ) crystals, respectively. By integrating the PLLA melting peak and using $91 \mathrm{~J} \mathrm{~g}^{-1}$ as the melting enthalpy value for $100 \%$ PLLA a form crystal, the crystallinity of PLLA can be estimated to be $67 \% 24$. To further exam the PLLA chain orientation in BCC, selected area electron diffraction (SAED) patterns on individual PLLA- $b$-PEG BCCs were recorded, and the results are shown in Fig. 2h. PLLA (200) and (110) diffraction spots are observed, suggesting the $c$-axis and the polymer chains are along the radial direction of the crystalsome. The diffraction spots are arc-shaped, which is attributed to the continuous lattice splay/distortion in order for the single crystal to fit into the curved nano-sized space $^{19,25}$. The SAED pattern therefore confirms the crystalsome shell is made of single crystal-like lamellae with polymer chains normal to the interface.

To further verify the shell thickness, AFM measurement was conducted on the small crystal pieces after breaking the BCCs by ultrasonication. AFM image and the corresponding crosssectional height profile (Fig. 3a) shows a total thickness of the shell is $\sim 4.5 \mathrm{~nm}$, including both PEG and PLLA layers. This is significantly thinner than the flat PLLA- $b$-PEG single crystals $(11.1 \pm 0.2 \mathrm{~nm}(n=10)$, supplementary Figure 3$)$. Based on the density and molecular weight of each segment, the thickness of PEG and PLLA layer can be estimated to be 2 and $2.5 \mathrm{~nm}$ respectively, which is consistent with the cryo-TEM image in Fig. 2c. As the polymer chain folds normal to the crystal surface, and using the molecular weight and the two-chain orthorhombic unit cell of PLLA $a$ form $(a=1.066 \mathrm{~nm}, b=0.616 \mathrm{~nm}$, and $c=2.888 \mathrm{~nm}$, Supplementary Note 1) with $10_{3}$ helical conformation, it can be calculated that each PLLA chain folds nine times, shown in Fig. 3b. The grafting density $\sigma$ of PEG on the surface can therefore be calculated to be $0.3 \mathrm{PEG}$ chain $\mathrm{nm}^{-2}$. Of interest is that PLLA crystallized into such thin lamellae, which leads to the nine-time fold of the polymer chain. In general polymer crystallization, the crystal thickness is determined by the surface free energy of the crystal folded surface, the heat of fusion, and undercooling of the crystallization process ${ }^{26-28}$. The present case is more complex. In addition to the above-mentioned parameters, entropy loss of the PEG brush upon PLLA crystallization, the molecular weights of the polymer, and the droplet size should all factor in determining the final thickness of the PLLA crystals.

On the basis of PEG chain grafting density, the distance between the anchoring points of the adjacent PEG chains, $D$, is $1.81 \mathrm{~nm}$ (See Supplementary Note 1 for calculation of chain folding number, grafting density and adjacent PEG distance). Flory radius of $5000 \mathrm{~g} \mathrm{~mol}^{-1}$ PEG in water can be calculated following $R_{F} \approx N^{3 / 5} a^{29}$, where $a, N$ and $R_{F}$ are defined as monomer size, the degree of polymerization and Flory radius. In the present case, $a=0.35 \mathrm{~nm}$ and $N=113^{30}$, and the calculated $R_{F}=5.95 \mathrm{~nm}$, much greater than the adjacent chain distance $1.81 \mathrm{~nm}^{29}$. Therefore, PEG layer in aqueous will take brush conformation, and the brush thickness $L$ can be calculated based on $L \approx N a^{(5 / 3)} D^{-(2 / 3) 29}$. Plugging in the above-mentioned values of $N, a$, and $D$, the calculated $L$ is $13.2 \mathrm{~nm}$, which is consistent with the cryo-TEM observation (Fig. 2c). Note that because the distance between the anchoring points of adjacent PEG is directed by PLLA crystallization, the PEG grafting density can be precisely controlled by PLLA chain folding and the lamellar thickness ${ }^{31,32}$. The spatial distribution of the brush molecules on the surface is extremely uniform due to the crystallization of PLLA, similar to our recently demonstrated polymer brush synthesis using polymer single crystals as the template ${ }^{33}$. This controlled uniform brush layer may improve the circulating performance of the BCCs, which is discussed in the following sections. We also anticipate that the grafting density can be further tuned by changing polymer molecular weights as well as the crystallization condition as previously reported $^{33}$, which will be the focus of our future work.

To understand how the PLLA chains crystallize near the liquid-liquid interface, we used molecular simulations to study 


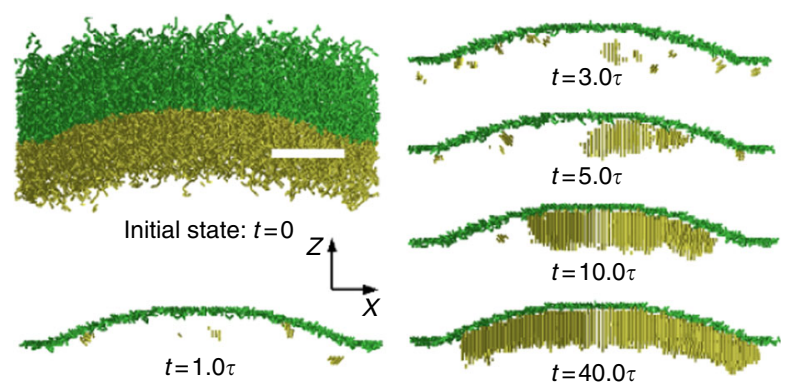

b

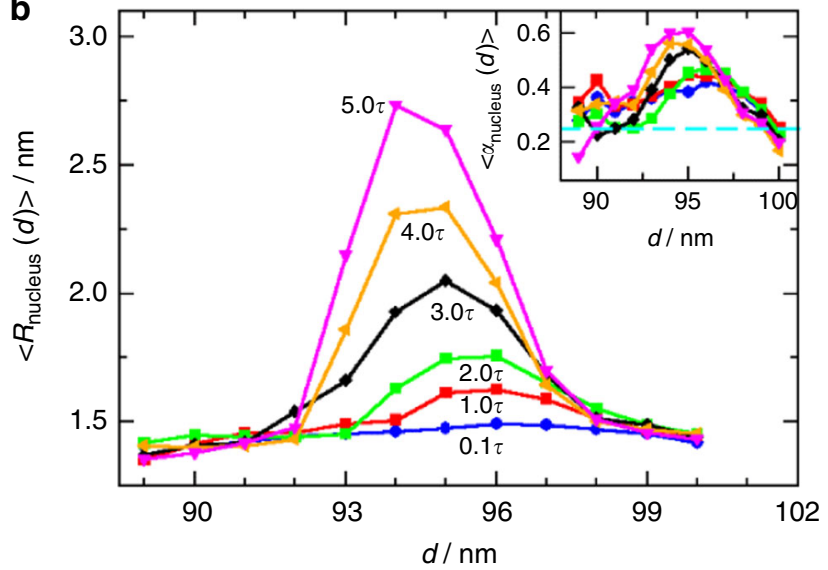

Fig. 4 Simulation study of the PLLA crystals grown near the curved liquid -liquid interface. a Snapshots of initial state for the PLLA- $b$-PEG chains near the curved interface and the crystallized PLLA bonds at $t=1.0 \tau, 3.0 \tau$, $5.0 \tau, 10.0 \tau$, and $40.0 \tau$. The snapshots are plotted in the range of $46 \mathrm{~nm}<y$ $<56 \mathrm{~nm}$, where $y=51 \mathrm{~nm}$ is the cross-section $x z$ plane through the droplet center. The yellow and green colors show the PLLA and PEG components correspondingly, and the green lines at different $t$ show the position of liquid-liquid interface. $\mathbf{b}$ Radial distributions of average nucleus size at different time $t=0.1 \tau, 1.0 \tau, 2.0 \tau, 3.0 \tau, 4.0 \tau$, and 5.0 $\tau$. Here the average is taken from 24 independent simulations, and the positions of nuclei/ crystals are determined by its center of mass (CM). Inset shows the radial distribution of average nuclei orientation at different time, with the same color coding as that in the main panel. The dashed cyan line in the inset indicates that the nuclei have no preferred orientation statistically (see Supplementary Note 2). Scale bar in a is $20 \mathrm{~nm}$

the nucleation kinetics (simulation details are in Supplementary Methods) ${ }^{34,35}$. Figure 4 a shows the snapshots of the initial PLLA$b$-PEG chains located at the curved interface, and the PLLA nuclei at different times, $t=1.0 \tau, 3.0 \tau, 5.0 \tau, 10.0 \tau$, and $40.0 \tau$ (here $\tau$ is the longest relaxation time of PLLA chains in solution ${ }^{36}$, Supplementary Figure 5). One can see that at $t=1.0 \tau$, there are some small PLLA nuclei formed near the interface. As time increases, these nuclei continue to grow, and some smaller ones dissolve or are merged into the growing nuclei (see the time evolution of average nucleus number and size in Supplementary Figure 6). Eventually only one single crystal is left in the system (see snapshots at $t=10.0 \tau$ and $40.0 \tau$ ).

To identify the accurate position where the nucleus grows, we calculate the radial distribution of the average nucleus size $R_{\text {nucleus }}(d)$ (see definitions in Supplementary Note 2), here $R_{\text {nucleus }}$ is the radius of a virtual spherical nucleus (see Supplementary Note 2) and $d$ is the distance between the center of mass (CM) of nucleus and the droplet center. Figure $4 \mathrm{~b}$ shows the variation $d$ of $R_{\text {nucleus }}(d)$ at different $t$. One can find that from $t=1.0 \tau$ to $5.0 \tau$, the nucleus grows at $d \cong 94.5 \sim 95.5 \mathrm{~nm}$, rather than from the
PLLA- $b$-PEG block junctions at the interface $(d \cong 98 \sim 100 \mathrm{~nm})$. This slightly off-interface growth can be understood by the radial distribution of average nuclei orientations (see inset of Fig. $4 \mathrm{~b}$ and definition in Supplementary Note 2). We found that the nuclei are preferred to be vertical to the interface at $d \cong 95 \mathrm{~nm}$, while near the interface $(d \cong 98 \sim 100 \mathrm{~nm})$, they have no preferred orientation. This can be explained as follows: the PLLA chains are stretched vertically to the curved interface due to the high grafting density $^{37}$, giving rise to a preferred nuclei orientation. However, this orientation would be suppressed near the liquid-liquid interface due to the influence of PEG and water solvents. Therefore, in average, the nuclei are grown in the droplet slightly off the liquid-liquid interface. Eventually, the crystal will cover the whole inner surface of the droplet ${ }^{38}$, forming a single crystallike crystalsome similar to Fig. 2e.

Mechanical property of PLLA-b-PEG BCCs. There are two major methods to characterize the mechanical property of polymer vesicles, namely micropipette aspiration technique and AFM-based nano-indentation ${ }^{39}$. Due to their small size, the mechanical property of PLLA- $b$-PEG BCCs was measured via AFM nano-indentation. In the experiment, aqueous solution of PLLA- $b$-PEG BCCs was drop casted onto a precleaned glass slide and dried under ambient condition. A crystalsome with smooth surface was chosen for the indentation experiment ${ }^{40,41}$. AFM image and the height profile of the crystalsome were acquired under Tapping Mode, as shown in Fig. 3c inset. The forcedeformation curve is shown in Fig. 3c, averaged from 14 times of indentation and the error bars represent standard deviation. After indentation, another AFM image was taken (Supplementary Figure 2) to confirm that the original BCC morphology was not deformed, indicating elastic deformation of the BCC during the indentation experiment. The slope of the fitted line of the deformation portion of the curve was used as the shell stiffness $k_{\text {shell }}$. The Young's modulus of the dry state PLLA- $b$-PEG shell can be calculated to be $11.5 \mathrm{GPa}^{42,43}$. The membrane bending modulus of the BCC shown in Fig. 3 in dry state can therefore be determined to be $9.8 \times 10^{-17} \mathrm{~J}$ (see Supplementary Note 3 for detailed calculation), which is significantly higher than polymersomes or crystalsomes. Note that in aqueous solution, PEG layer would be solvated and will not significantly affect the BCC bending modulus. Therefore the bending modulus of BCCs shell in water can be estimated to be $K_{\text {bend,wet }}=3.63 \times 10^{-17} \mathrm{~J}$ (see Supplementary Note 3 for the calculation). Table 1 summarizes the typical values of sizes and mechanical properties of selected liposomes, polymersomes, homopolymer crystalsomes, BCCs, and viral capsids. It is clear that PLLA- $b$-PEG BCCs have about 140-8000 times higher bending modulus than polymersomes with much greater thickness, even with a much thinner shell thickness. Thus, BCCs shell would be more stable and more robust under shear flow, suggesting they may provide better protection for the potential cargo inside. Interestingly, BCC shares many features with viral capsid: they are spherical, mechanically strong and resilient. The typical thickness of the viral capsid is between 2 and $5 \mathrm{~nm}$, which is also very close to the present BCC structure, suggesting that BCC might provide an ideal self-assembled capsid-mimic system for desired applications such as drug carriers.

In vivo circulation and biodistribution studies of BCCs. To test the feasibility of using BCCs for intravenous delivery, in vivo circulation experiments were carried out. A hydrophobic fluorescent dye, 1,1'-dioctadecyl-3,3,3',3'-tetramethylindodicarbocyanine, 4chlorobenzenesulfonate salt $(\mathrm{DiD})$ was encapsulated in these BCCs during the crystal growth. DiD has been cited as a marker 
Table 1 A comparison of mechanical characteristics among liposomes, polymersomes, crystalsomes, and viral capsids.

\begin{tabular}{|c|c|c|c|c|c|}
\hline \multicolumn{2}{|c|}{ Self-assembled structures } & \multirow{3}{*}{$\frac{\text { Diameter (nm) }}{37.0 \pm 7.9}$} & \multirow{3}{*}{ Thickness (nm) } & \multirow{2}{*}{$\begin{array}{l}\text { Young's modulus (MPa) } \\
2 \pm 0.8\end{array}$} & \multirow{2}{*}{$\begin{array}{l}\text { Bending Modulus }\left(k_{\mathrm{B}} T\right) \\
7\end{array}$} \\
\hline Liposomes & EggPC $^{59}$ & & & & \\
\hline & DPPC60 & & & 81 & 275 \\
\hline Polymersomes & $\mathrm{PDMS}_{68}-b-\mathrm{PMOXA}_{11} 39$ & 306 & $16 \pm 2$ & $17 \pm 11$ & $1703 \pm 1216$ \\
\hline Crystalsomes & PLLA crystalsomes ${ }^{19}$ & 224 & 22.5 & 4160 & $1.1 \times 10^{6}$ \\
\hline & PLLA-b-PEG BCCs & $\sim 180$ & $4.5 \pm 0.4$ & $(1.1 \pm 0.2) \times 10^{4}$ & $(2.4 \pm 0.8) \times 10^{5}($ dry $)$ \\
\hline Viral capsids & Hepatitis $B^{61}$ & 14.2 & 2.24 & 0.26 & 70.5 \\
\hline
\end{tabular}

EggPC egg yolk phosphatidylchlolin, DPPC dipalmitoylphosphatidylcholine, PDMS-b-PMOXA(dimethylsiloxane)-block-poly(2-methyloxazoline), PEO-b-PEE Poly(ethylene oxide)-b-poly(ethylethylene), MLV murine leukemia virus

aExpansion modulus
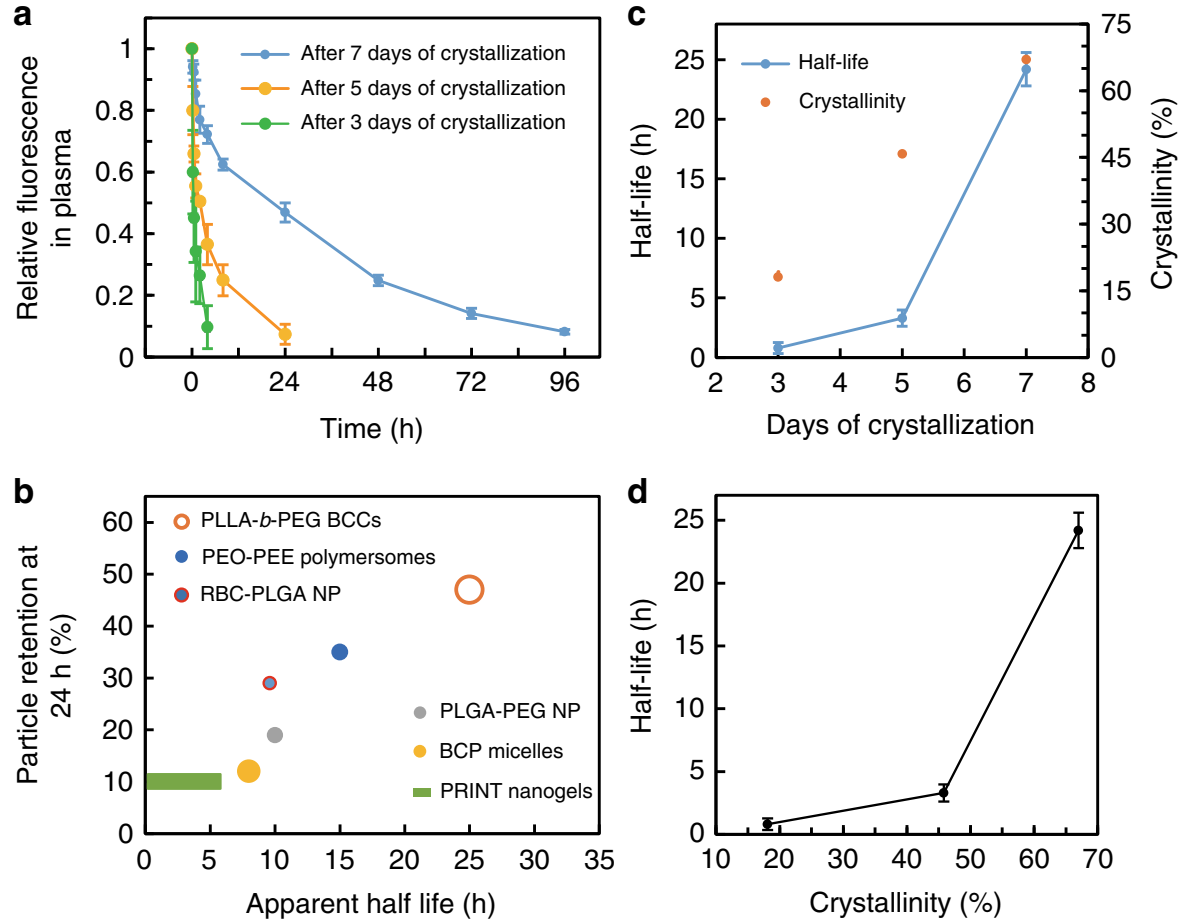

Fig. 5 Dependence of BCC blood circulation on their crystallinity. a In vivo circulation of DiD-loaded BCCs crystallized for different times. $\mathbf{b}$ In vivo circulation performance among representative polymeric nanoparticles (PEO- $b$-PEE polymersomes ${ }^{47}$, RBC-PLGA NP6, surface-modified PLGA-PEG NP44, $\mathrm{BCP}$ micelles ${ }^{56}$, and PRINT nanogels ${ }^{30}$ ) with long blood circulation. A plot of relative intensities at $24 \mathrm{~h}$ vs. apparent half-lives, where relative symbol sizes represent relative hydrodynamic sizes/shapes and data are taken from the respective references. $\mathbf{c}$ BCC crystallinity and half-life variation with different crystallization times. d The correlation of circulation half-life and crystallinity of BCCs. Error bars in $\mathbf{a}, \mathbf{c}$ represent mean \pm s.d, $n=3-5$

in a number of blood circulation studies for polymeric nanoparticles $^{6,44}$. An in vitro control experiment was firstly conducted to confirm if BCCs are leaky. To this end, DiD-encapsulated PLLA- $b$-PEG BCCs were dispersed in a PBS solution supplemented with $10 \%$ fetal bovine serum (FBS). At different time intervals, BCCs were collected, and fluorescent intensity was measured. It was shown that there was minimal fluorescence signal decay (2\%) after 5 days of incubation (Supplementary Discussion, Supplementary Figure 8), indicating BCCs are well sealed and nearly impermeable for DiD in this experimental condition. The sealed structure was further demonstrated by the slow release of hydrophilic nitrobenzoxadiazole (NBD)based dye $(\sim 1.7 \%$ per $24 \mathrm{~h}$, Supplementary Figure 9$)$. In a separate experiment, BCCs also showed negligible intake of hydrophilic 5-Carboxyfluorescein after $24 \mathrm{~h}$ incubation (Supplementary Figure 10). In our in vivo experiments, DiDencapsulated BCCs with an average size of $200 \mathrm{~nm}$ in saline were systemically administered into $\mathrm{BALB} / \mathrm{c}$ mice through tail vein injection at a dose of $1 \mathrm{mg}$ per mouse. A series of blood samples were collected at various time points post injection. The circulation profiles were obtained by measuring the fluorescence intensity of the sample plasma (Fig. 5a). The blue curve shows the circulation performance of the BCCs crystallized for 7 days. The fluorescence intensity decay follows a classical onecompartment PK model, yielding a long circulation half-life of $24.2 \pm 1.4 \mathrm{~h}$ (mean \pm s.d., $n=5)$. Remarkably, there are 47,14 and $8 \%$ of BCCs remained in the blood after 24,72 and $96 \mathrm{~h}$ circulation, respectively.

Most polymeric nanoparticles administered intravenously are quickly cleared by the mononuclear phagocyte system $^{45,46}$. Numerous strategies have been reported to improve nanoparticle blood circulation time. Nanoparticle size, surface PEGylation and mechanical properties have been shown to affect nanoparticle circulation 45,46 . Figure $5 \mathrm{~b}$ summarizes the blood circulation 
apparent half-life and particle retention at $24 \mathrm{~h}$ for several reported nanoparticle systems with long circulation lives. Here the apparent half-life is defined as the time for the nanoparticle concentration to fall to half of the original value, and the percentage particle retention at $24 \mathrm{~h}$ denotes the fraction of particles retained in plasma $24 \mathrm{~h}$ post injection. For example, the reported spherical Poly(ethylene oxide)- $b$-poly(ethylethylene) (PEO-b-PEE) polymersome has an apparent half-life $15 \mathrm{~h}$ with a $24 \mathrm{~h}$ retention of $\sim 35 \%{ }^{47}$. Perry et al. reported that with a relatively low PEG brush grafting density, soft hydrogel PRINT nanoparticles showed a half-life $3 \mathrm{~h}$ and a $24 \mathrm{~h}$ retention of $10 \%{ }^{30}$. $\mathrm{Hu}$ et al. coated RBC membranes onto PLGA nanoparticles to bypass in vivo clearance, reaching a $9.6 \mathrm{~h}$ apparent half-life with $29 \%$ retention at $24 \mathrm{~h}^{6}$.

Compared to the above-mentioned leading polymeric nanoparticle carriers, BCCs show an impressive half-life of $24.2 \mathrm{~h}$ and a $24 \mathrm{~h}$ retention of $47 \%$. Furthermore, most nanoparticles investigated in these previous studies are rather small with an average size of $90-100 \mathrm{~nm}$ (except for PRINT nanoparticle, which has a dimension of $80 \times 80 \times 320 \mathrm{~nm})$. It is known that particle size is a key factor for long circulation. Larger nanoparticles of 171 and $243 \mathrm{~nm}$ were reported to get cleared in blood twice as fast as that of smaller nanoparticles $(80 \mathrm{~nm})^{46,48}$. Given the relative large size of the BCCs, it is even more intriguing to observe the long circulating time value of the present system. Furthermore, previous studies showed that polymer crystallization reduced blood circulation of polymersomes and cylindrical polymer brushes, which is apparently not the case in BCCs ${ }^{15,49}$. To better understand the mechanism of the observed long circulation time of BCCs, we investigated the circulation behaviors of BCCs that were crystallized for shorter periods of time, e.g. 3 days and 5 days. The results are also plotted in Fig. 5a, which shows that BCCs of shorter crystallization time (3-day and 5-day) exhibit much faster clearance in the blood yet 5-day crystallization time yields a blood retention better than 3-day crystallization. Fitted with the one-compartment model, BCCs after 3-day and 5-day crystallizations have half-lives of $0.80 \pm 0.46 \mathrm{~h}(n=4)$ and $3.3 \pm 0.68 \mathrm{~h}(n=3)$, respectively. Figure $5 \mathrm{c}$ summarizes the change of half-lives with increasing crystallization time. DLS measurement (Supplementary Figure 11a) confirmed that all the BCCs have similar hydrodynamic radii and size distribution. Supplementary Figure $11 \mathrm{c}$, d demonstrate that morphologies of 3-day and 5-day crystallized BCCs also maintained capsule-like structures with similar particle sizes. Interestingly, crystallinity calculated from DSC heating thermograms (Supplementary Figure 11b) illustrated shorter crystallization time rendering lower crystallinity (18.1, 45.8 and $67 \%$ for 3, 5, and 7 days of crystallization, respectively), which is also plotted in Fig. 5c. Combining all these results, we can conclude that there is a close correlation between the BCC crystallinity and the blood circulation time, shown in Fig. $5 \mathrm{~d}$. The ultra-long circulation time of the 7-day crystallized BCCs arises from the high crystallinity and uniform crystalline chain packing on the curved BCC shells which minimize defect formation in the crystals. In 3-day, 5-day crystallized BCCs and the previously reported polymersome cases, relatively poorly controlled crystals lead to defect-rich particles, which may be prone to disassembly under blood flow. Furthermore, the long circulation may also be facilitated by the uniform PEG brushes formed by crystallization-induced brush-formation. As we recently reported, BCP crystallization-templated polymer brushes are highly tunable and extremely uniform polymer brushes with minimal defects. However, in reported nanoparticle circulation studies, PEG layers are typically formed by graftingto methods, and are not uniform due to the steric hindrance of the macromolecular chemistry ${ }^{33}$.
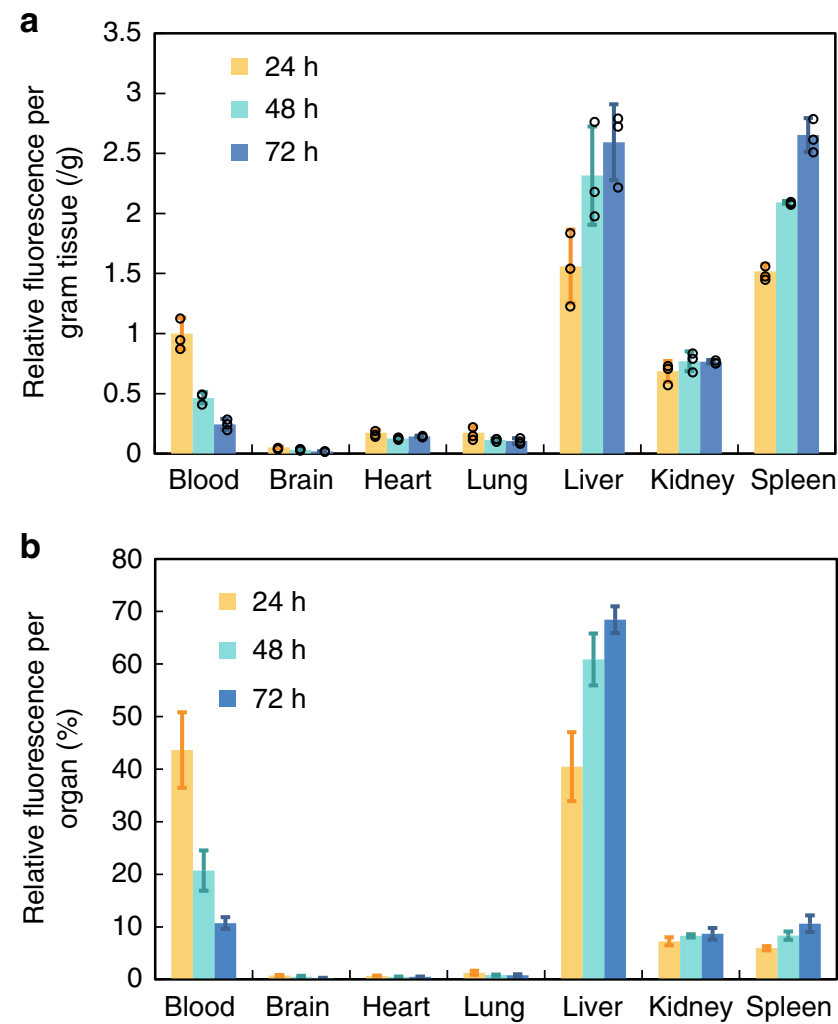

Fig. 6 Biodistribution in mice of BCCs after 7 days of crystallization. At different time points (24, 48, and $72 \mathrm{~h}$ ), fluorescence intensity of homogenized tissues were measured. a Fluorescence intensity per gram of tissue. Individual data points are shown in circles. b Relative fluorescence signal per organ. Error bars, mean \pm s.d, $n=3$

Nanoparticle distribution in organs was investigated for up to 3 days via IVIS imaging and homogenization (Fig. 6a, b). The relative numbers of PLLA- $b$-PEG BCCs in the blood over 3 days were consistent with the circulation data presented in Fig. 5a that the amount of BCCs in the blood was halved every day, which follows the one-compartment model formula $C(t)=C\left(\boldsymbol{t}_{0}\right) \cdot e^{-k \cdot t_{1 / 2} 50}$. With respect to distributions in other organs, the BCCs primarily accumulated to liver and spleen, which are organs of the reticuloendothelial system (RES). Thus, BCCs were mainly sequestered by the liver in such a time frame, which is consistent with the recent understanding on how hard nanomaterials are cleared by the liver ${ }^{51}$. The PLLA- $b$-PEG BCCs accumulation in the liver and spleen increased with time, suggesting that the blood fluorescence mainly arises from the dyes in BCCs rather than the leakage of the dye. In the latter case, the dye would be secreted by kidneys, leading to the decrease of fluorescence signal from the liver 6 .

Polymeric vesicles have been shown to accumulate in the liver ${ }^{47}$. The uptake of nanoparticles by hepatic macrophages, Kupffer cells may be reduced when the liver-specific opsonin absorption is minimal ${ }^{52}$. Besides liver, the PLLA- $b$-PEG BCCs exhibited a high accumulation in the spleen up to $72 \mathrm{~h}$. Feijen et al. reported that polymersomes with a size of $95 \mathrm{~nm}$ primarily accumulated in the liver whereas maintained a very low uptake amount in the spleen from 24 to $72 \mathrm{~h}^{53}$. PLLA- $b$-PEG BCCs interestingly showed a primary and increasing accumulation over time in the spleen probably due to their relatively larger sizes. It was reported that nanoparticles with size beyond $150 \mathrm{~nm}$ are more likely to get entrapped within the liver and spleen ${ }^{54}$ Large stealth liposomes $(>200 \mathrm{~nm})$ also showed a significant 
accumulation in the spleen due to the mechanical filtration in addition to phagocytosis ${ }^{55}$. Application of nanocarriers has long been restricted by the available choices of particle size, while the optimal size was considered to be $70-150 \mathrm{~nm}^{54,56}$. PLLA- $b$-PEG BCCs showed that large particles with the size of $\sim 200 \mathrm{~nm}$ circulated up to $96 \mathrm{~h}$ in the blood, which opens the door for medical applications of large nanoparticles $(\sim 200 \mathrm{~nm})$. Furthermore, the hollow structure is advantageous for potential delivery applications since (1) it provides space to encapsulate a large number of drugs at a level that is difficult to achieve by other drug carriers; (2) minimal amount of polymer materials is used in a capsule; and (3) these hollow capsules also provide large surface area to mass ratio. Future research will be focused on better controlling the structure, morphology, size distribution and surface function of the BCCs for targeted applications.

\section{Discussion}

In conclusion, we demonstrated the preparation of robust BCCs for long blood circulation. The emulsion-solution crystallization method is efficient to guide polymer chain folding into uniform crystalline packing at curved liquid/liquid interface to form single crystal-like structure. As a BCP ensemble, BCCs exhibit superior mechanical stability, suggesting that tailoring crystallization is a route to prepare robust $\mathrm{BCP}$ vesicles. In vivo circulation tests demonstrate that the BCCs can circulate up to $96 \mathrm{~h}$ in the blood with a half-life of $24.2 \pm 1.4 \mathrm{~h}$, and $47 \%$ retention at $24 \mathrm{~h}$ post injection. This excellent circulation performance was attributed to controlled crystallization of PLLA and the resultant uniform PEG brush layer on the BCC surfaces. Our observation is different to the previous perception that crystallization reduces polymersome circulation, providing a strategy to generate long circulating nanomaterials. We envisage that the hollow, robust, tunable BCCs could lead to a new class of nanoparticle carriers for drug delivery and gene therapy.

\section{Methods \\ Materials and animals. Poly (L-lactide)-block-poly (ethylene glycol) (PLLA-b PEG, $M_{n}=6000-5000 \mathrm{~g} \mathrm{~mol}^{-1}$ ) was purchased from Polymer Source Inc. 1,1- dioctadecyl-3,3,3,3-tetramethylindocarbocy amine perchlorate (DiD oil) was pur- chased from Life Technologies. Toluene and $\mathrm{NaCl}$ were purchased from Sigma Aldrich. All materials were used as received. Female BALB/c mice were purchased from Charles River Laboratory.}

Preparation of PLLA-b-PEG block copolymer crystalsomes (BCCs). $22 \mathrm{mg}$ PLLA- $b$-PEG and $12 \mu \mathrm{g}$ of fluorescent dye (DiD) were first dissolved in $0.4 \mathrm{~g}$ toluene in a glass tube. Then, $16 \mathrm{~g}$ DI water was added into the above polymer solution at $95^{\circ} \mathrm{C}$. After emulsification at $95^{\circ} \mathrm{C}$ for $2 \mathrm{~min}$ via probe sonication, the emulsion was kept at $95^{\circ} \mathrm{C}$ for another $30 \mathrm{~s}$ before quenched to $25^{\circ} \mathrm{C}$ for crystallization. After crystallization for 3-9 days, the solution was dialyzed against DI water through $50 \mathrm{~nm}$ membranes at $25^{\circ} \mathrm{C}$ for $4-6 \mathrm{~h}$, and the procedure was repeated for five times before the sample was collected through a $0.45 \mu \mathrm{m}$ syringe filter.

Before in vivo study, the above-mentioned BCCs aqueous solution was further dialyzed again saline $(0.9 \mathrm{w} / \mathrm{v} \% \mathrm{NaCl}$ aqueous solution) overnight and then concentrated with $30 \mathrm{~K}$ molecular weight cutoff (MWCO) Amicon Ultra-4 Centrifugal filters. Gas chromatography experiments showed no detectable amount of toluene in the BCC samples (See Supplementary Methods, Supplementary Figures 12, 13).

Characterization of PLLA-b-PEG BCCs. Hydrodynamic radius were measured by DLS using Zetasizer Nano ZS90. SEM images were taken on a ZEISS Supra 50VP microscope with a $1 \mathrm{kV}$ accelerating voltage. To prepare SEM samples, a drop of $10 \mu \mathrm{l}$ BCCs aqueous solution was cast onto cover slides and then dried under vacuum overnight. Before SEM imaging, the sample was coated with $\mathrm{Pt} / \mathrm{Pd}$.

TEM bright field imaging and SAED experiments were conducted using a JEOL JEM2100 microscope with a $120 \mathrm{kV}$ accelerating voltage. TEM samples were prepared by drop casting BCCs' aqueous solution onto a carbon-coated $\mathrm{Cu}$ grid. Cryogenic-transmission electron microscopy (cryo-TEM) was performed on a FEI Talos F200C TEM (University of Delaware, DE). A vitrified sample was prepared with an FEI Vitrobot apparatus ${ }^{57,58}$. The vitrified samples were transferred to a cryoholder in a sample stage immersed in liquid nitrogen. The cryoholder was kept below $-170{ }^{\circ} \mathrm{C}$ during the imaging to prevent sublimation. The digital images were recorded by a Talos F200C TEM equipped with a FEI Falcon direct electron detector.

AFM images and force spectra were acquired using a Bruker Dimension Icon AFM equipped with a tapping mode and PeakForce mode. Samples were dried on piranha-cleaned cover slide. The cantilever used is Bruker TESPA with tip height of $10-15 \mu \mathrm{m}$, and tip radius of $8 \mathrm{~nm}$. The spring constant of the cantilever is $2.030 \mathrm{~N} \mathrm{~m}^{-1}$, calibrated by the thermal tune method. DSC experiments were conducted on DSC Q2000 with Tzero pans from TA Instruments with a $10^{\circ} \mathrm{C} \mathrm{min}^{-1}$ heating rate.

Monte Carlo simulation. We use a coarse-grained lattice model to study the nucleation kinetics of PLLA chains near the curved liquid-liquid interface. The PLLA- $b$-PEG chain is modeled as a diblock copolymer of $A_{38} B_{28}$, with A and $B$ corresponding to the PEG and PLLA monomers, and the total monomers in one chain is $N=66$. The curved liquid-liquid interface is set up in prior in the simulation box of $100 \times 100 \times 99 \mathrm{~nm}^{3}$, then PLLA- $b$-PEG chains are relaxed in this interface. The total chain number is chosen as $n=3000$, calculated from the experimental grafting density 0.3 chains per $\mathrm{nm}^{2}$. The polymer chains are moved via a micro-relaxation model ${ }^{34}$, which allows each segment to change positions with its neighboring solvent sites, accompanied by the sliding diffusion along the chain direction if necessary ${ }^{35}$. Conventional metropolis sampling was employed in each micro-relaxation step. The reduced temperature is set as $k_{\mathrm{B}} T / E_{c}=3.6$, and other parameters are summarized in Supplementary Note 2. Information.

In vitro release study. PLLA- $b$-PEG BCCs labeled with $\mathrm{DiD}$ was dispersed in a $10 \%$ FBS PBS solution. The mixture was shaken at $100 \mathrm{rpm}$ at $37^{\circ} \mathrm{C}$. At each time interval, BCCs were collected with an MWCO $100 \mathrm{~K}$ (Amicon Ultra-4) tube with centrifugation. Equal volume of $10 \%$ FBS in PBS was used to resuspend BCCs. The fluorescent intensity was measured at an excitation/emission wavelength of $600 / 665 \mathrm{~nm}$ by a microplate reader (Infinite M200, TECAN) and compared with the initial intensity for quantification.

In vivo circulation study. PLLA- $b$-PEG BCCs labeled with $\mathrm{DiD}$ in saline was systemically administered through tail vein injection. Each female BALB/c mouse was slowly injected with $100 \mu \mathrm{L} 10 \mathrm{mg} \mathrm{mL}^{-1}$ of NP solution. A small volume of $10 \mu \mathrm{L}$ of blood was collected at $2 \mathrm{~min}, 15 \mathrm{~min}, 0.5 \mathrm{~h}, 1 \mathrm{~h}, 2 \mathrm{~h}, 4 \mathrm{~h}, 8 \mathrm{~h}$, $24 \mathrm{~h}, 48 \mathrm{~h}$ and up to $96 \mathrm{~h}$ post i.v. injection. The blood was diluted in $200 \mu \mathrm{L}$ of heparin/PBS $\left(16 \mathrm{U} \mathrm{mL}^{-1}\right)$ solution to avoid blood coagulation. The samples were centrifuged at $300 \times g$ for $5 \mathrm{~min}$ to remove blood cells, and then $180 \mu \mathrm{L}$ of the supernatant was collected for measurement at excitation/emission wavelength of $600 / 665 \mathrm{~nm}$ by a microplate reader (Infinite M200, TECAN). One-compartment pharmacokinetics model was used to calculate in vivo circulation half-lives via PKSolver ${ }^{49}$. All animal procedures were conducted according to the protocols of the Committee on Animal Care of Drexel in compliance with NIH guidelines.

In vivo biodistribution study. Female Balb/c mice at 8 weeks old were randomly separated into three groups and were i.v. injected with $150 \mu \mathrm{L}$ of DiD-labeled PLLA- $b$-PEG BCCs at $10 \mathrm{mg} \mathrm{mL}^{-1}$ on days 1,2 , and 3 respectively. The control group was injected with the same amount of saline. On day 4 , all the mice were sacrificed and their brains, lungs, hearts, livers, spleens, kidneys as well as blood were collected. To quantify the amount of PLLA- $b$-PEG BCCs in each organ, all the organs were weighted and homogenized. The fluorescence intensities of DiD-containing homogenized solution were detected at an excitation/emission wavelength of $600 / 665 \mathrm{~nm}$ by a microplate reader (Infinite M200, TECAN).

Data availability. The data are available from the corresponding author upon reasonable request.

Code availability. The simulation code is available from the corresponding author upon request.

Received: 14 November 2017 Accepted: 2 July 2018

Published online: 01 August 2018

\section{References}

1. Noguchi, H. \& Gompper, G. Shape transitions of fluid vesicles and red blood cells in capillary flows. Proc. Natl. Acad. Sci. USA 102, 14159-14164 (2005).

2. Michel, J. P. et al. Nanoindentation studies of full and empty viral capsids and the effects of capsid protein mutations on elasticity and strength. Proc. Natl. Acad. Sci. USA 103, 6184-6189 (2006).

3. Ivanovska, I. L. et al. Bacteriophage capsids: tough nanoshells with complex elastic properties. Proc. Natl Acad. Sci. USA 101, 7600-7605 (2004). 
4. Merkel, T. J. et al. Using mechanobiological mimicry of red blood cells to extend circulation times of hydrogel microparticles. Proc. Natl Acad. Sci. USA 108, 586-591 (2011).

5. Rodriguez, P. L. et al. Minimal "self" peptides that inhibit phagocytic clearance and enhance delivery of nanoparticles. Science 339, 971-975 (2013).

6. Hu, C. M. J. et al. Erythrocyte membrane-camouflaged polymeric nanoparticles as a biomimetic delivery platform. Proc. Natl Acad. Sci. USA 108, 10980-10985 (2011).

7. $\mathrm{Xu}, \mathrm{L}$. et al. Systemic tumor-targeted gene delivery by anti-transferrin receptor scFv-immunoliposomes. Mol. Cancer Ther. 1, 337-346 (2002).

8. Akhtar, S. \& Benter, I. F. Nonviral delivery of synthetic siRNAs in vivo. J. Clin. Invest. 117, 3623-3632 (2007).

9. Discher, B. M. et al. Polymersomes: tough vesicles made from diblock copolymers. Science 284, 1143-1146 (1999).

10. Discher, D. E. \& Eisenberg, A. Polymer vesicles. Science 297, 967-973 (2002).

11. O’Reilly, R. K., Hawker, C. J. \& Wooley, K. L. Cross-linked block copolymer micelles: functional nanostructures of great potential and versatility. Chem. Soc. Rev. 35, 1068-1083 (2006).

12. Ahmed, F., Hategan, A., Discher, D. E. \& Discher, B. M. Block copolymer assemblies with cross-link stabilization: from single-component monolayers to bilayer blends with PEO- PLA. Langmuir 19, 6505-6511 (2003).

13. Vilgis, T. \& Halperin, A. Aggregation of coil crystalline block copolymersequilibrium crystallization. Macromolecules 24, 2090-2095 (1991).

14. Van Horn, R. M. et al. Solution crystallization behavior of crystalline -crystalline diblock copolymers of poly(ethylene oxide)-block-poly $(\varepsilon$ caprolactone). Macromolecules 43, 6113-6119 (2010).

15. Rajagopal, K. et al. Curvature-coupled hydration of semicrystalline polymer amphiphiles yields flexible worm micelles but favors rigid vesicles: polycaprolactone-based block copolymers. Macromolecules 43, 9736-9746 (2010)

16. Meng, G., Paulose, J., Nelson, D. R. \& Manoharan, V. N. Elastic instability of a crystal growing on a curved surface. Surf. Sci. 343, 634-637 (2014)

17. Garcia, N. A., Pezzutti, A. D., Register, R. A., Vega, D. A. \& Gomez, L. R. Defect formation and coarsening in hexagonal 2D curved crystals. Soft Matter 11, 898-907 (2015)

18. Bausch, A. R. et al. Grain boundary scars and spherical crystallography. Science 299, 1716-1718 (2003).

19. Wang, W. et al. Highly robust crystalsome via directed polymer crystallization at curved liquid/liquid interface. Nat. Commun. 7, 10599 (2016).

20. Katre, N. V. The conjugation of proteins with polyethylene glycol and other polymers: altering properties of proteins to enhance their therapeutic potential. Adv. Drug Deliv. Rev. 10, 91-114 (1993).

21. Staub, M. C. \& Li, C. Y. Confined and directed polymer crystallization at curved liquid/liquid interface, Macrom. Chem. Phys. 219, 1700455 (2018)

22. Ahmed, F. \& Discher, D. E. Self-porating polymersomes of PEG-PLA and PEG-PCL: hydrolysis-triggered controlled release vesicles. J. Control Rel. 96, 37-53 (2004)

23. Cheng, S., Smith, D. M. \& Li, C. Y. How does nanoscale crystalline structure affect ion transport in solid polymer electrolytes? Macromolecules 47, 3978-3986 (2014).

24. Wunderlich, B. Thermal Analysis of Polymeric Materials (Springer, Berlin Heidelberg, 2005).

25. Li, C. Y. et al. Double twist in helical polymer "soft" crystals. Phys. Rev. Lett. 83, 4558-4561 (1999).

26. Wunderlich B. Macromolecular Physics (Academic Press, New York, 1976).

27. $\mathrm{Li}, \mathrm{B}$. \& Li, C. Y. Immobilizing Au nanoparticles with polymer single crystals, patterning and asymmetric functionalization. J. Am. Chem. Soc. 129, 12-13 (2007).

28. Li, C. Y. Polymer single crystal meets nanoparticles. J. Poly. Sci. Poly. Phys. 47, 2436-2440 (2009).

29. De Gennes, P. G. Conformations of polymers attached to an interface. Macromolecules 13, 1069-1075 (1980).

30. Perry, J. L. et al. PEGylated PRINT nanoparticles: the impact of PEG density on protein binding, macrophage association, biodistribution, and pharmacokinetics. Nano Lett. 12, 5304-5310 (2012).

31. Chen, W. Y. et al. Onset of tethered chain overcrowding. Phys. Rev. Lett. 93, 028301 (2004).

32. Xiong, $\mathrm{H}$. et al. Scrolled polymer single crystals driven by unbalanced surface stresses: rational design and experimental evidence. Macromolecules $\mathbf{4 4}$ 7758-7766 (2011).

33. Zhou, T., Qi, H., Han, L., Barbash, D. \& Li, C. Y. Towards controlled polymer brushes via a self-assembly-assisted-grafting-to approach. Nat. Commun. 7, 11119 (2016)

34. Hu, W. \& Frenkel, D. Polymer crystallization driven by anisotropic interactions. In Interphases and Mesophases in Polymer Crystallization III (eds Allegra, G.) (Springer, Berlin, Heidelberg, 2005).

35. Tang, Q., Hu, W. \& Napolitano, S. Slowing down of accelerated structural relaxation in ultrathin polymer films. Phys. Rev. Lett. 112, 148306 (2014).
36. Müller, M. \& Daoulas, K. C. Single-chain dynamics in a homogeneous melt and a lamellar microphase: a comparison between Smart Monte Carlo dynamics, slithering-snake dynamics, and slip-link dynamics. J. Chem. Phys. 129, 164906 (2008).

37. Zha, L. \& Hu, W. Molecular simulations of confined crystallization in the microdomains of diblock copolymers. Prog. Polym. Sci. 54, 232-258 (2016).

38. Hu, W. The physics of polymer chain-folding. Phys. Rep https://doi.org/ 10.1016/j.physrep.2018.04.004 (2018).

39. Jaskiewicz, K., Makowski, M., Kappl, M., Landfester, K. \& Kroeger, A. Mechanical properties of poly(dimethylsiloxane)-block-poly(2methyloxazoline) polymersomes probed by atomic force microscopy. Langmuir 28, 12629-12636 (2012).

40. Vaziri, A. \& Mahadevan, L. Localized and extended deformations of elastic shells. Proc. Natl Acad. Sci. USA 105, 7913-7918 (2008).

41. Vaziri, A. Mechanics of highly deformed elastic shells. Thin Wall Struct. 47, 692-700 (2009).

42. Reissner, E. Stresses and small displacements of shallow spherical shells. I. J. Math. Phys. 25, 80-85 (1946).

43. Wang, W. D., Staub, M. C., Zhou, T., Smith, D. M., Qi, H., Laird, E. D., Cheng, S. \& Li, C. Y. Polyethylene nano crystalsomes formed at a curved liquid/liquid interface, Nanoscale 10, 268-276 (2018).

44. Zhou, H. et al. Hyaluronidase embedded in nanocarrier PEG shell for enhanced tumor penetration and highly efficient antitumor efficacy. Nano Lett. 16, 3268-3277 (2016).

45. Hrkach, J. et al. Preclinical development and clinical translation of a PSMAtargeted docetaxel nanoparticle with a differentiated pharmacological profile. Sci. Transl. Med. 4, $128 \mathrm{ra39}$ (2012).

46. Alexis, F., Pridgen, E., Molnar, L. K. \& Farokhzad, O. C. Factors affecting the clearance and biodistribution of polymeric nanoparticles. Mol. Pharm. 5, 505-515 (2008).

47. Photos, P. J., Bacakova, L., Discher, B., Bates, F. S. \& Discher, D. E. Polymer vesicles in vivo: correlations with PEG molecular weight. J. Control Rel. 90, 323-334 (2003).

48. Fang, C. et al. In vivo tumor targeting of tumor necrosis factor-alpha-loaded stealth nanoparticles: effect of MePEG molecular weight and particle size. Eur. J. Pharm. Sci. 27, 27-36 (2006)

49. Mullner, M. et al. Size and rigidity of cylindrical polymer brushes dictate long circulating properties in vivo. ACS Nano 9, 1294-1304 (2015).

50. Zhang, Y., Huo, M., Zhou, J. \& Xie, S. PKSolver: an add-in program for pharmacokinetic and pharmacodynamic data analysis in Microsoft Excel. Comput. Methods Prog. Biomed. 99, 306-314 (2010).

51. Tsoi, K. M. et al. Mechanism of hard-nanomaterial clearance by the liver. Nat. Mater. 15, 1212-1221 (2016).

52. Stolnik, S., Illum, L. \& Davis, S. S. Long circulating microparticulate drug carriers. Adv. Drug Deliv. Rev. 64, 290-301 (2012).

53. Lee, J. S. et al. Circulation kinetics and biodistribution of dual-labeled polymersomes with modulated surface charge in tumor-bearing mice: comparison with stealth liposomes. J. Control Rel. 155, 282-288 (2011).

54. Blanco, E., Shen, H. \& Ferrari, M. Principles of nanoparticle design for overcoming biological barriers to drug delivery. Nat. Biotechnol. 33, 941-951 (2015).

55. Moghimi, S. M., Hedeman, H., Muir, I. S., Illum, L. \& Davis, S. S. An investigation of the filtration capacity and the fate of large filtered stericallystabilized microspheres in rat spleen. Biochim. Biophys. Acta 1157, 233-240 (1993).

56. Wang, J. et al. The role of micelle size in tumor accumulation, penetration, and treatment. ACS Nano 9, 7195-7206 (2015).

57. Ni, B. et al. Pathway toward large two-dimensional hexagonally patterned colloidal nanosheets in solution. J. Am. Chem. Soc. 137, 1392-1395 (2015).

58. Percec, V. et al. Modular synthesis of amphiphilic Janus glycodendrimers and their self-assembly into glycodendrimersomes and other complex architectures with bioactivity to biomedically relevant lectins. J. Am. Chem. Soc. 135, 9055-9077 (2013).

59. Liang, X., Mao, G. \& Simon, Ng. K. Y. Probing small unilamellar EggPC vesicles on mica surface by atomic force microscopy. Colloid Surf. B 34, 41-51 (2004).

60. Park, J. W. Sulfatide incorporation effect on mechanical properties of vesicles. Colloid Surf. B 80, 59-62 (2010).

61. Roos, W. H., Bruinsma, R. \& Wuite, G. J. L. Physical virology. Nat. Phys. 6, 733-743 (2010)

62. Kol, N. et al. Mechanical properties of murine leukemia virus particles: effect of maturation. Biophys. J. 91, 767-774 (2006).

\section{Acknowledgements}

This work was supported by the National Science Foundation Grant DMR-1308958 and DMR 1709136. C.Y.L. and H.C. are grateful for the support of Drexel DARE and PA Department of Health, CURE grant programs. H.C. acknowledges the support from National Institute of Allergy and Infectious Diseases of the National Institutes of Health 
under Award Number R21AI133372. Q.T. acknowledges the financial support from the Deutsche Forschungsgemeinschaft under grant Mu1674/15-1. The simulations are performed at GWDG Göttingen.

\section{Author contributions}

H.Q., H.Z., H.C. and C.Y.L. designed the research; H.Q., T.Z., S.M., S.K. and M.C.S. fabricated and characterized crystalsomes; J.Y.L. and D.J.P. performed the cryo-TEM work; H.Q. and L.H. conducted the AFM indentation measurements; Q.T. and W.H. performed the simulations; H.Z., Z.F. and H.C. conducted the in vivo circulation and biodistribution study. H.Q., H.Z., H.C. and C.Y.L. wrote the paper.

\section{Additional information}

Supplementary Information accompanies this paper at https://doi.org/10.1038/s41467018-05396-x.

Competing interests: The authors declare no competing interests.

Reprints and permission information is available online at http://npg.nature.com/ reprintsandpermissions/
Publisher's note: Springer Nature remains neutral with regard to jurisdictional claims in published maps and institutional affiliations.

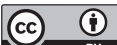

Open Access This article is licensed under a Creative Commons Attribution 4.0 International License, which permits use, sharing, adaptation, distribution and reproduction in any medium or format, as long as you give appropriate credit to the original author(s) and the source, provide a link to the Creative Commons license, and indicate if changes were made. The images or other third party material in this article are included in the article's Creative Commons license, unless indicated otherwise in a credit line to the material. If material is not included in the article's Creative Commons license and your intended use is not permitted by statutory regulation or exceeds the permitted use, you will need to obtain permission directly from the copyright holder. To view a copy of this license, visit http://creativecommons.org/ licenses/by/4.0/.

(C) The Author(s) 2018 